\title{
Die Krise und die sozialen Sicherungssysteme
}

\author{
Heiner Ganßmann \\ Ralf K. Himmelreicher
}

Ein deutlicher Rückgang des Sozialprodukts, ansteigende Beschäftigungsverluste und ein drastisches Anwachsen der Staatsverschuldung sind in Deutschland die Hauptfolgen der globalen Wirtschaftskrise. Wie wird die neu gewählte Bundesregierung dieses problematische Dreieck auflösen? Die diskutierte Eindämmung der Staatsschulden - womöglich noch bei Steuersenkungen - wäre ohne Kürzungen der Sozialleistungen nicht denkbar. Doch selbst wenn die Sozialleistungen bei wachsenden Defiziten auf dem Status quo gehalten werden, sind viele Arbeitslose und geringfügig Beschäftigte langfristig von Altersarmut bedroht. Die Krisenlasten würden als Einkommensverluste und unzureichende Altersversorgung vordringlich bei Unterbeschäftigten und gering Entlohnten landen.

\section{1}

Vor dem Hintergrund der aktuellen Finanz- und Wirtschaftskrise diskutieren wir, inwiefern das System der sozialen Sicherung und die Versicherten von der Krise betroffen sind. Unser Thema ist spekulativ, weil wir nicht wissen können, wie sich die gegenwärtige Krise, die nicht mehr nur eine Finanzkrise ist, weiter entwickelt. Wir wissen derzeit auch nicht, wie weit die aktuellen Versuche greifen, die Auswirkungen der Krise mittels politischer Interventionen einzudämmen. Worauf wir aber zurückgreifen können, sind, erstens, Erfahrungswerte aus bisherigen Krisenverläufen und, zweitens, bereits beobachtbare Auswirkungen der Krise auf Stand und Entwicklung der sozialen Sicherungssysteme. Und schließlich kennen wir, drittens, die Interessenlagen in Bezug auf soziale Sicherungssysteme: Wer will was, wie viel, von wem, wozu? Wir gehen im Folgenden auf diese drei Aspekte ein und ziehen daraus Schlussfolgerungen über mögliche oder wahrscheinliche Konflikte um die Zukunft der sozialen Sicherungssysteme.

\section{Krisenablauf}

\subsection{HISTORISCHE ERFAHRUNGEN}

Die folgenden Vermutungen über den weiteren Krisenverlauf stützen sich auf die historischen Krisenvergleiche von Reinhart/ Rogoff (2009). Sie haben die mit Finanzkrisen verbundenen wirtschaftlichen Entwicklungsverläufe im Hinblick auf Dauer und Tiefe der Rückgänge verglichen. Dabei stellen sie allerdings, was die Aufklärung über die gegenwärtige Situation begrenzt, nicht in Rechnung, dass es sich bei der derzeitigen Krise um einen global, zumindest zeitweise synchron ablaufenden Prozess handelt. Die Krise begann im Sommer 2007 zwar als Bankenkrise in den USA, sie hat aber 2008 über den stark globalisierten Finanzsektor hinaus die gesamte Weltwirtschaft erfasst. Das hatte den - gerade für Exportländer wie Deutschland - relevanten Effekt, dass auch nationale Wirtschaften, die weder einen Immobilienboom noch besonders spekulative Finanzmärkte hatten, schwer betroffen sind. Einerseits muss man also befürchten, dass die wirtschaftliche Erholung wegen der global synchronen Krise schwieriger wird als bei den von Reinhart/Rogoff verglichenen partiellen Krisen. Andererseits beanspruchen Zentralbanken und Wirtschaftspolitik, aus vergangenen Krisen gelernt zu haben und rechtzeitig und ausreichend gegenzusteuern. Der Vergleich der aktuellen mit vorausgegangenen Krisen ergibt also nur Anhaltspunkte für die vermutliche Entwicklung. Jedoch sind gerade frühere Bankenkrisen in reichen Ländern ein interessanter Orientierungspunkt, um den bevorstehenden, wahrscheinlichen Verlauf in Deutschland einschätzen zu können. Reinhart/ Rogoff sprechen hier von The Big Five Crises in Spanien (1977), Norwegen (1987), Finnland (1991), Schweden (1991) und Japan (1992).

Diese Krisen haben typische ${ }^{1}$ Verlaufsformen bezüglich Dauer der Depression und Erholungszeiten. Das bedeutet für

(1) Finanzmärkte: Die Aktienkurse fallen um durchschnittlich $55 \%$ im Laufe von drei bis vier Jahren. Der obere Wendepunkt liegt ein Jahr vor der Krise. Nach dem Ein- bruch vergehen zwei bis drei Jahre, bis der vorherige Höhepunkt übertroffen wird. Der V-förmige Verlauf zeigt eine relativ schnelle Erholung an.

(2) Immobilienmärkte: Die Häuserpreise gehen um 30-50\% (Durchschnitt $35 \%$ ) zurück; die Depression dauert über sechs Jahre.

(3) Gütermärkte: Der durchschnittliche Rückgang des realen Sozialprodukts pro Kopf beträgt über $9 \%$, aber die Dauer des Rückgangs ist mit ca. zwei Jahren kürzer als auf dem Arbeitsmarkt.

(4) Arbeitsmärkte: Die Arbeitslosenrate steigt über vier bis fünf Jahre bis auf ein Niveau, das um durchschnittlich sieben Prozentpunkte über dem vor der Krise liegt.

(5) Staatsverschuldung: Infolge der Krise bleiben die Staatseinnahmen bis zu drei Jahre lang unter dem Vorkrisenniveau,

\section{Wir folgen der Charakterisierung mithilfe arithme- tischer Mittel.}

\footnotetext{
Heiner Ganßmann, Prof. Dr., Hochschullehrer i. R. für Soziologie an der Freien Universität Berlin. Arbeitsschwerpunkte: Wechselwirkungen zwischen Sozialstaat und Arbeitsmarkt, Soziologie des Geldes. e-mail: heiner.ganssmann@googlemail.com Ralf K. Himmelreicher, PD Dr., Referent im Forschungsdatenzentrum der Rentenversicherung (www.FDZ-RV.de) Berlin und Privatdozent am Institut für Soziologie der Freien Universität Berlin. Arbeitsschwerpunkte: Einkommen und Vermögen im Lebenslauf, Verteilungsfragen, empirische Wirtschafts- und Sozialforschung. e-mail: ralf.himmelreicher@drv-bund.de
} 
während gleichzeitig die Staatsschulden stark ansteigen und sich in den drei Jahren nach der Krise fast verdoppeln (kumulatives Wachstum um $86 \%$ ).

\subsection{MÖGLICHE FOLGEN FÜR DIE ENTWICKLUNG IN DEUTSCHLAND}

Nehmen wir an, die Entwicklung in Deutschland würde - mit einigen Modifikationen, z. B. wegen der vergleichsweise stabilen Immobilienpreise oder infolge der Exportlastigkeit der deutschen Wirtschaft diesem typischen Verlauf folgen. Das bedeutete

(1) für die Arbeitslosigkeit: Der Ausgangswert der Arbeitslosigkeit war in Deutschland im internationalen Vergleich sehr hoch. Die Arbeitslosenrate lag im Oktober/ November 2008 bei $8 \%$. Wenn man den Durchschnittszuwachs von Reinhart/Rogoff zugrunde legt, würde sie in den Jahren bis 2013 infolge der Krise auf etwa $15 \%$ ansteigen;

(2) für die Einkommen: Bei durchschnittlichem Krisenverlauf würde das reale Sozialprodukt pro Kopf in diesem und im nächsten Jahr um etwa $9 \%$ zurückgehen. Aktuell wird für 2009 ein Rückgang um 5,4 \% oder sogar weniger prognostiziert. Doch selbst bei derart optimistischen Werten schrumpft nicht nur der Verteilungsspielraum, sondern es wird darum gehen, wer die Einkommensverluste trägt. Die Löhne werden bei steigender Arbeitslosigkeit bestenfalls stagnieren. $\mathrm{Ob}$ sie auch real sinken, hängt davon $\mathrm{ab}$, wieweit sich eine Deflationsspirale entwickelt. Für eine Erholung müssen - in der üblichen Reihenfolge - zuerst die Gewinne wieder steigen, dann folgen die Löhne, dann erst die Sozialleistungen;

(3) für den Staat: Die Staatseinnahmen würden bis etwa 2011 unter dem Niveau vor der Krise bleiben. Infolgedessen müsste die Verschuldung unabhängig von der eingeschlagenen wirtschaftspolitischen Linie massiv ansteigen, nicht nur wegen der Ausputzkosten für die Not leidenden „systemrelevanten" Banken, sondern vor allem, weil die Staatseinnahmen hinter den krisenbedingt ansteigenden Ausgaben zurückbleiben. Der Druck wächst, entweder die Steuern zu erhöhen oder die Ausgaben, also vor allem die Sozialleistungen, zu senken.

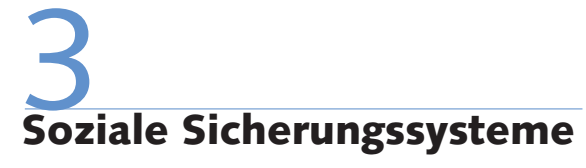

Sozialversicherungen können in Krisenzeiten mit hoher Verschuldung wegen ihrer antizyklischen Ausgestaltung als „automatische Stabilisatoren" der Nachfrage funktionieren. Dennoch unterliegen sie einer Budgetrestriktion, der „Konsumbeschränkung", wie Mackenroth (1952) das genannt hat: Quelle für sozialstaatliche Leistungen ist immer das laufende reale Volkseinkommen, ${ }^{2}$ gleichgültig, wie persönliche Ansprüche auf einen Anteil daran erworben wurden (z. B. über kapitalgedeckte oder umlagefinanzierte Rentensysteme). Das bedeutet, dass das Schicksal der Sozialstaatsklienten systemisch eng mit dem der Lohneinkommensbezieher verkoppelt ist, und zwar nicht nur über das Zusammenspiel von Erwerbsarbeit, Geldleistungen und Sicherungsansprüchen, sondern grundsätzlich dadurch, dass die Lohneinkommen (inklusive der sogenannten Arbeitgeberanteile) die Hauptquelle für die Finanzierung sozialstaatlicher Leistungen sind.

Für die Einschätzung der möglichen Entwicklungen der sozialen Sicherungssysteme ist deshalb die Entwicklung der Lohnquote ausschlaggebend. Sie nimmt in Deutschland im Trend seit Jahrzehnten genauer seit 1982 - außergewöhnlich stark ab (Brenke 2009, S. 557f.), abgesehen von zyklischen Schwankungen: In Krisen brechen die Gewinne schneller ein als die Löhne. Nach einem Zwischenhoch aufgrund

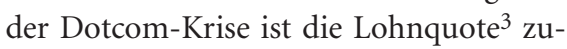
letzt von $67 \%$ auf $61 \%$ gefallen. Für die sozialen Sicherungssysteme bedeutet das, dass ihre herkömmliche Finanzierungsbasis schrumpft. Die erwartbare Beschäftigungskrise wird diese Finanzierungsprobleme verschärfen, weil mehr Personen von der Leistungs- auf die Empfängerseite wandern.

Vor dem Hintergrund der genannten Rahmenbedingungen diskutieren wir im Folgenden, inwiefern das System der sozialen Sicherung und seine Versicherten von der aktuellen Finanz- und Wirtschaftskrise betroffen sein können. Da sich das deutsche Sozialsystem aus einer Fülle von Einrichtungen mit unterschiedlichen Leistungen für verschiedene Personengruppen zusammensetzt, konzentrieren wir uns auf zwei zentrale Elemente: die Arbeitslosenversicherung und die Altersvorsorge für ab- hängig, genauer: für sozialversicherungspflichtig Beschäftigte. Aus institutioneller Sicht stehen damit die Finanzierung und die Leistungen der Bundesagentur für Arbeit (BA) (Abschnitt 3.1), der gesetzlichen Rentenversicherung (GRV) sowie die betriebliche Altersvorsorge (BAV) und die teilweise staatlich geförderte private Altersvorsorge (PAV) (Abschnitt 3.2) im Zentrum der Analyse. ${ }^{4}$ Aus der Perspektive des Lebensverlaufs liegen die Risiken, arbeitslos oder Empfänger von Kurzarbeitergeld zu werden, zeitlich gesehen vor den mit dem Alter verbundenen Risiken einer Unterversorgung, die vom vorgängigen Erwerbsverlauf beeinflusst werden. Wir betrachten deshalb zunächst die unmittelbaren Auswirkungen der Arbeitslosigkeit.

\subsection{SOZIALE SICHERUNG BEI ARBEITSLOSIGKEIT}

Grundsätzlich beeinflusst die wirtschaftliche Entwicklung, vermittelt über den Arbeitsmarkt, die Systeme sozialer Sicherung, weil der Arbeitsmarkt als Transmissionsriemen zwischen konjunkturellem Verlauf und sozialer Absicherung wirkt (Sesselmeier et al. 2009, S. 183). Insofern hängen die Folgen der Finanz- und Wirtschaftskrise für die BA und ihre Versicherten vor allem von Dauer und Ausmaß der Krise und der davon bestimmten Entwicklung der Anzahl der Beschäftigten und deren Bezahlung ab. Erste Folgen der Wirtschaftskrise auf dem Arbeitsmarkt sind unübersehbar. Dem Präsidenten der BA, Frank-J. Weise, zufolge, sind "die bisherigen Auswirkungen des Abschwungs aber vergleichsweise moderat; vor allem die starke Nutzung der Kurzarbeit stabilisiert den Arbeitsmarkt" (BA 2009a).

Die Subventionierung von Kurzarbeit ist ein wichtiges Instrument zur Abfederung konjunkturell bedingter Arbeitsausfälle.

2 Abgesehen von der Möglichkeit, auch Ansprüche an das Sozialprodukt anderer Länder zu erwerben. Damit kann man aber nur bedingt ein Sicherheitsversprechen - und darum geht es sozialpolitisch verbinden, wie die Eigentümer von allerhand Wertpapieren in der Finanzkrise kürzlich erleben mussten.

3 Bereinigt um die Verschiebung des Anteils der abhängig Beschäftigten an allen Erwerbstätigen.

4 Damit werden u. a. Leistungen und Maßnahmen der gesetzlichen und privaten Krankenversicherung, der gesetzlichen Unfallversicherung und Maßnahmen der gesetzlichen Pflegeversicherung nicht betrachtet. 
Bisher beschränkte sich die Dauer des Bezugs von sogenannter konjunktureller Kurzarbeit ${ }^{5}$ auf sechs Monate. Durch das Konjunkturpaket II wurde die Bezugsdauer auf 18 Monate erhöht. Im Rahmen einer weiteren Änderung können Betriebe nun „Kurzarbeitergeld Plus“ 24 Monate in Anspruch nehmen. Diese Regelung wird auch auf Betriebe ausgedehnt, die bereits 2008 Kurzarbeit gefahren haben (Schaff 2009; vgl. hierzu auch Deeke 2009).

So hat sich die Anzahl der Kurzarbeiter im März 2009 gegenüber dem Vorjahr in Westdeutschland um 951.000 auf 1.064 .000 erhöht. In Ostdeutschland stieg die Zahl von 135.000 auf 177.000 an. Der durchschnittliche Arbeitszeitausfall aller Kurzarbeiter betrug im März 2009 knapp $35 \%$. Für durchschnittliche Kurzarbeiter bedeutet dies, dass ihre pauschalierten Nettolöhne, bestehend aus Lohn für gekürzte Stundenzahl plus Kurzarbeitergeld, rund $14 \%$ (ohne Kinder) bzw. 11 \% (mit Kindern) geringer ausfallen als zuvor. ${ }^{6}$

Im Juni gingen bei der BA neue Anzeigen für 204.000 Kurzarbeiter aus konjunkturellen Gründen ein. Erste Schätzungen für den Juli signalisierten neue Anzeigen für rund 175.000 Personen (BA 2009b). Die BA hat angekündigt, dass sie die Mittel für Kurzarbeit im Jahr 2009 von den ursprünglich veranschlagten 300 Mio. $€$ auf 2,1 Mrd. $€$ aufstocken wird. In Vollzeitäquivalente umgerechnet reduziert das Kurzarbeitergeld die Anzahl der Neuzugänge in Arbeitslosengeld (ALG) I um rund 500.000 Personen. Ein kurzfristiger bzw. ein bis zu 24 Monate andauernder Entlastungseffekt ist damit sicherlich zu verzeichnen. Empfänger von Kurzarbeit stellen sich in der Regel besser als Bezieher von ALG I und leisten höhere Sozialversicherungsbeiträge. Während des Bezugs von Kurzarbeitergeld werden Beiträge auf der Basis des reduzierten Verdienstes des Beschäftigten gezahlt und paritätisch vom Versicherten und Arbeitgeber getragen. Vom Arbeitgeber zusätzlich gezahlt werden Beiträge auf der Basis von $80 \%$ des wegen Kurzarbeit ausgefallenen Verdienstes.

Der Vorteil von Kurzarbeit ist die stabilisierende Wirkung auf die Beschäftigung und Einkommen in der Krise. Allerdings fließen die Mittel überproportional häufig in Großbetriebe, insbesondere in die Automobilindustrie und deren Zulieferbetriebe und in die Bauwirtschaft, sodass insgesamt vor allem Branchen mit einem hohen Anteil männlicher Beschäftigter davon profi- tieren. Damit unterstützt die Subventionierung von Kurzarbeit vor allem bereits gut gesicherte „Insider“, obgleich das Kurzarbeitergeld von allen Beitragszahlern in der Arbeitslosenversicherung finanziert wird. Es findet ein Transfer aus dem Dienstleistungssektor hin zum verarbeitenden Gewerbe und zur Bauwirtschaft statt. Für die Bezieher ergibt sich eine sozialpolitisch bewirkte Glättung ihres Einkommensstromes.

Die Verlängerung der Bezugsdauer von Kurzarbeitergeld und des ALG I auf bis zu drei bzw. vier Jahre für ältere Beschäftigte erhöht die Ausgaben der BA. Hinzu kommt eine weitere Ausgabensteigerung beim Insolvenzgeld, das die BA Arbeitnehmern für drei Monate zahlt. Im Mai 2009 meldeten die deutschen Amtsgerichte 2.663 Unternehmensinsolvenzen, eine Steigerung um $14,9 \%$ gegenüber Mai 2008 (Statistisches Bundesamt 2009). Auf der Einnahmeseite macht sich die aktuelle Schwäche der Gesamtwirtschaft als - im Juli 2009 noch gemäßigter - Rückgang der Anzahl der Erwerbstätigen bemerkbar.

Zusammenfassend lässt sich vor dem Hintergrund der Absenkung des Beitragssatzes zur Arbeitslosenversicherung zum 1. Januar 2009 um 0,5 Prozentpunkte auf 2,8 \% festhalten: Aufgrund des konjunkturellen Abschwungs sanken die Einnahmen der BA im ersten Quartal 2009 auf 6,3 Mrd. €, bei Ausgaben in Höhe von 10,4 Mrd. $€$ im gleichen Zeitraum (Statistisches Bundesamt 2009). Damit dürften die Rücklagen der BA, Ende 2008 noch 16,7 Mrd. $€$, innerhalb eines Jahres zum größten Teil wieder in den Arbeitsmarkt zurückfließen (BA 2009c).

\subsection{SOZIALE SICHERUNG IM ALTER}

Beschäftigungsverluste und sinkende Arbeitsvolumen infolge der Finanz- und vor allem Wirtschaftskrise wirken sich auf die Sparfähigkeit der Personen im erwerbsfähigen Alter wie auch auf die Leistungsansprüche der Erwerbstätigen und vor allem der Rentnerinnen und Rentner aus. Grundsätzlich sind Ansprüche oder Anwartschaften gegenüber GRV, BAV und PAV mit unterschiedlichen Risiken verbunden, wobei sich Finanzkrisen am stärksten auf die Höhe der Prämien von Neurentnern, also die aktuellen Rentenzugänge auswirken. Vor allem die Alterseinkünfte aus der PAV, insbesondere bei fondsbasierten Verträgen, können direkt und kurzfristig beeinflusst werden.
Die OECD-Studie „Pensions at a Glance" (Stand Mai 2009) kommt zu dem Ergebnis, dass alle OECD-Länder die Folgen der Krise spüren werden, unabhängig davon, ob die Altersvorsorge hauptsächlich auf einem kapitalgedeckten oder einem umlagefinanzierten System beruht. Besonders stark betroffen sind jedoch jene Länder, in denen die Altersvorsorge häufig über kapitalgedeckte Pensionsfonds mit hohen Aktienanteilen läuft (OECD 2009).

Angesichts der in Deutschland politisch gewollten Umgewichtung hin zu einer stärkeren Rolle der betrieblichen und privaten Vorsorge in der Alterssicherung stellt sich deshalb insbesondere die Frage, wie sich die Wirtschafts- und Finanzkrise auf kapitalgedeckte Formen der Alterssicherung auswirkt. Grundsätzlich ist dabei zu beachten, dass die GRV vielfältige „Elemente des sozialen Ausgleichs" enthält. Betriebliche Altersversorgung hingegen orientiert sich auch an betriebswirtschaftlichen Zielen und wird zudem personalpolitisch als Anreizinstrument eingesetzt. Demgegenüber ist private Altersvorsorge auf Rentabilität und Beitragsäquivalenz ausgerichtet, d. h. Beiträge und Leistungen stehen in einem finanzmathematisch kalkulierten engen Verhältnis zueinander. Der sogenannte Paradigmenwechsel (Schmähl 2000) verändert die Gewichte dieser Bestandteile des Alterssicherungssystems, mit Folgen für die Einkommenssituation der verschiedenen Bevölkerungsgruppen, für die Rentabilität von Aufwendungen zur Altersvorsorge, für die Bindung an den Arbeitgeber, für die Risiken sowie schließlich für die Erfüllung sozialpolitischer Ziele wie Reduzierung von Ungleichheit oder Vermeidung von Altersarmut (Viebrok et al. 2004). Während früher der GRV politisch das Ziel der Lebensstandardsicherung zugeschrieben wurde und das Ziel der Armutsvermeidung im Alter primär von der Sozialhilfe bzw. der Grundsicherung im Alter erreicht werden sollte, wird heute eine stärkere ergänzende Vorsorge durch BAV

\footnotetext{
5 Für Saison-Kurzarbeitergeld und Transferkurzarbeitergeld gelten teilweise abweichende Regelungen, die hier nicht dargestellt sind.

6 Als Ausgleich für den Lohn, der durch die Kürzung der Stundenzahl entfällt, erhalten Arbeitnehmer Kurzarbeitergeld. Es beträgt analog zum Arbeitslosengeld (ALG) I bei Arbeitnehmern ohne Kinder $60 \%$ des ausfallenden pauschal festgestellten Nettolohnes. Arbeitnehmer, die Kinder zu unterhalten haben, erhalten 67 \% (Schaff 2009, S. 13).
} 
und PAV angestrebt, um die Ausgaben und damit den Beitragssatz der Rentenversicherung zu reduzieren.

\section{AUSWIRKUNGEN AUF DIE GRV}

Bei der gesetzlichen Rentenversicherung (GRV) ist im ersten Quartal 2009 - bei konstantem Beitragssatz - ein Einnahmezuwachs um $1,9 \%$ auf 58,6 Mrd. $€$ zu verzeichnen. Dem stand ein ebenfalls leichter Zuwachs der Ausgaben um $2 \%$ auf 60,0 Mrd. $€$ gegenüber. Im Vergleich zum ersten Quartal des Vorjahres erhöhte sich das Finanzierungsdefizit der GRV dadurch nur gering (von 1,3 auf 1,4 Mrd. €, vgl. Statistisches Bundesamt 2009). Durch Krisenreaktionen wie Abbau von Überstunden, Reduktion von Urlaubsansprüchen und Ausgleich von Arbeitszeitkonten hat sich die Einnahmesituation der GRV kaum verändert. Bei Bezug von Kurzarbeitergeld reduzieren sich die individuellen Pflichtbeiträge proportional zum Verdienstausfall und betragen damit selbst bei „Kurzarbeit null“ $80 \%$ des vorherigen Niveaus. Bei einem durchschnittlichen Arbeitszeitausfall von $35 \%$ reduzieren sich die GRV-Pflichtbeiträge nur um rund sieben Prozentpunkte. Schließt sich an das Kurzarbeitergeld der Bezug von ALG I an, dann bezahlt die BA auf der Basis von $80 \%$ des zuletzt erzielten Einkommens den Rentenbeitrag von 19,9 \% an die GRV. Erst wenn die Beschäftigung stark zurückgeht und zugleich die Zahl der ALG-II-Empfänger deutlich steigt, wird die Krise auch in der GRV deutlich spürbar werden.

Für Bezieher von Arbeitslosengeld II zahlt die BA Rentenversicherungsbeiträge auf Basis eines beitragspflichtigen Entgelts von $205 €(\$ 166$ Abs. 1 Ziff. 2a SGB VI), also unabhängig von der tatsächlichen Höhe des Arbeitslosengeldes II. Für ein Jahr Bezug von Arbeitslosengeld II ergibt sich nach Werten von 2007 eine monatliche Rentenanwartschaft in Höhe von 2,19 €. Im Fall einer krisenbedingten Erhöhung des ALGII-Bezugs würden neben geringeren Einnahmen auch individuelle Anwartschaften gegenüber der GRV und in der Regel auch gegenüber privater und betrieblicher $\mathrm{Al}$ tersvorsorge erheblich sinken, weshalb in Abhängigkeit von der Dauer der Krise und den jeweiligen Beschäftigungschancen nach der Krise das Risiko von Altersarmut steigt.

Insgesamt sind die bisher zu beobachtenden Folgen der Krise auf die Einnahmesituation der GRV eher gering. Die
Kombination aus Kurzarbeitergeld und ALG I verschiebt drastische Einnahmeverluste um rund drei Jahre. Beschäftigungsabbau und steigende Langzeitarbeitslosigkeit bei gleichzeitiger krisenbedingter Lohnzurückhaltung können die Einkommenssituation in einigen Jahren zwar verschlechtern. Jedoch führt das am 19.06. 2009 beschlossene „Rentengarantiegesetz“ dazu, dass es selbst bei sinkenden Löhnen und Gehältern nicht zu Rentenkürzungen kommt. Vor dem Hintergrund sinkender Einnahmen und garantierter Ausgaben sind demgemäß Defizite im Haushalt der GRV erwartbar. Sie könnten die Erhöhung der Bundeszuschüsse und/oder des Beitragssatzes sowie Leistungskürzungen, etwa „Nullrunden“ während des Aufschwungs oder gekürzte Hinterbliebenenrenten, nach sich ziehen.

Wie sich das Leistungsniveau, d. h. die Höhe der Anwartschaften der Versicherten, bis vor der Krise entwickelt hat, lässt sich anhand der Entwicklung und Verteilung der Summe der persönlichen Entgeltpunk$\mathrm{te}^{7}$ von Neurentnerinnen und -rentnern im Zeitraum 1993-2007 beobachten (Himmelreicher/Stuchlik 2008, S. 543ff.):

Für männliche Rentner zeigt sich ein Rückgang ihrer Entgeltpunkte (gemessen am Median) um knapp $7 \%$ (auf 44) in den alten und um rund $18 \%$ (auf 42) in den neuen Bundesländern. Zudem ist eine $\mathrm{Zu}$ nahme der Streuung bei den Anwartschaften zu verzeichnen.

Bei Frauen zeigt sich ein anderes Bild: Neurentnerinnen in den alten Bundesländern erzielten 2007 rund $22 \%$ mehr Entgeltpunkte als noch 1993. Allerdings erfolgt diese Zunahme einseitig zugunsten von Bezieherinnen hoher und höchster Altersrenten und vollzieht sich auf einem insgesamt niedrigen absoluten Niveau von 14 Entgeltpunkten (Median). Die Entwicklung der Entgeltpunkte weiblicher Rentenzugangskohorten in den neuen Bundesländern hat sich wie folgt verändert: 2007 sanken die Anwartschaften um rund $6 \%$ auf durchschnittliche 29 Entgeltpunkte.

Insgesamt betrachtet ist in den zurückliegenden 15 Jahren eine starke Zunahme der Spreizung der Altersrenten zu beobachten. Diese Entwicklung der Entgeltpunkte lässt sich neben zunehmend perforierten Erwerbsbiografien mit Beschäftigungslücken vor allem am Ende der Erwerbsphase auf die Wirkung von Abschlägen bei vorzeitigem Rentenbeginn zurückführen: Im Jahr 2007 sind in den neuen Bundesländern mehr als $60 \%$ und in den alten mehr als $40 \%$ der Männer und Frauen von Abschlägen betroffen.

Die empirischen Befunde deuten auf einige Risiken hin: Im Zeitverlauf sinkende GRV-Anwartschaften spiegeln geringere Löhne in Relation zum durchschnittlichen Bruttoeinkommen in der Vergangenheit und/oder kürzere Erwerbsbiografien (mit Abschlägen) wider und damit auch reduzierte Ansparmöglichkeiten für zusätzliche Vorsorge - ein Teufelskreis, angesichts der zunehmenden Bedeutung der privaten und betrieblichen Alterssicherung. Nur lange Erwerbsbiografien mit hinreichend hohem sozialversicherungspflichtigen Einkommen führen zu ausreichender Altersversorgung. Selbst vergleichsweise kurze Phasen von Arbeitslosigkeit (ein bis drei Jahre) in der Erwerbsbiografie führen zu Einbußen der GRV-Anwartschaften von rund $10 \%$ (Riedmüller/Willert 2008). Insbesondere im Zusammenhang mit staatlich gesteuerten Anreizen zur Eigenvorsorge bestehen damit Risiken für diejenigen, die lange arbeitslos oder spät in die GRV eingestiegen sind, was etwa für Migranten zutrifft. Auch die zunehmende Lohnspreizung oder der Ausbau des Niedriglohnsektors sind angesichts der Befunde problematisch (Brenke 2007). Geringe Einkommen über längere Erwerbsphasen führen sowohl zu niedrigen GRV-Anwartschaften als auch zu geringerer Sparfähigkeit.

\section{AUSWIRKUNGEN AUF DIE BAV}

Die aktuelle Finanz- und Wirtschaftskrise beeinträchtigt direkt auch die betriebliche Altersversorgung (BAV). Durch den Einbruch der Kapitalmärkte Ende 2008 sind die Pensionsvermögen der DAX-Unternehmen um 13 Mrd. $€$ auf $125 \mathrm{Mrd}$. $€$ geschrumpft. In diesem Zusammenhang

\footnotetext{
7 Bei den im Folgenden dargestellten Befunden werden monatliche persönliche Entgeltpunkte der Versicherten dargestellt, unter individueller Berücksichtigung des Rentenartfaktors und des Zugangsfaktors. Diese so berechnete Summe der persönlichen Entgeltpunkte kann mit dem aktuellen Rentenwert multipliziert werden, um näherungsweise die Höhe der monatlichen Altersrente zu ermitteln. Entgeltpunkte sind eine relative Größe, bei der der individuelle Lohn durch den jeweiligen Durchschnittslohn dividiert wurde, weshalb langjährige Zeitvergleiche möglich werden. Der aktuelle Rentenwert (monatliche Altersrente für ein Kalenderjahr Durchschnittsverdienst) beträgt seit 01.07. 2009 monatlich $27,20 €$ (West) und $24,13 €$ (Ost).
} 
können in der betrieblichen Altersversorgung Finanzierungsprobleme erwartet werden. ${ }^{8}$ Vor allem bei der Entgeltumwandlung wirkt sich die mit der Wirtschaftskrise in Deutschland verbreitete Kurzarbeit aus. Reduzierte oder nicht mehr zahlbare Beiträge erhöhen die Versorgungslücke und können den oftmals in die BAV integrierten Schutz gegen finanzielle Folgen einer Berufsunfähigkeit gefährden. Ein Ausweg für Arbeitnehmer besteht darin, die Altersversorgung mit eigenen Beiträgen fortzuführen. Bei arbeitgeberfinanzierten Versorgungssystemen hingegen hat die Kurzarbeit nur dann Auswirkungen, wenn die Leistung einen Bezug zum versorgungsfähigen Einkommen hat. Arbeitgeber tragen im Fall von Leistungszusagen das Risiko, für zugesagte Leistungen einstehen zu müssen. In diesem Zusammenhang versuchen manche Unternehmen, Leistungszusagen zu reduzieren. $\mathrm{Zu}$ dem fallen Kosten für die Sicherung der BAV gegen Unternehmensinsolvenzen an. Somit können Wertverluste der Finanzanlage sowie mögliche Kosten für deren Insolvenzsicherung direkt zu Renditeminderungen oder zu reduzierten Renditeerwartungen und damit zu Akzeptanzproblemen gegenüber der BAV führen.

\section{AUSWIRKUNGEN AUF DIE PAV}

Die Verbreitung der staatlich geförderten privaten Altersvorsorge (PAV) hat deutlich zugenommen: Ende des ersten Quartals 2009 gab es 12,4 Mio. sogenannte RiesterVerträge, das sind knapp 300.000 mehr als im Vorjahr. Wenn man jedoch bedenkt, dass das Potenzial der Riester-berechtigten Personen auf zwischen 30 bis 36 Mio. Personen geschätzt wird (Corneo et al. 2007, S. 7), zeigt sich, dass die Quote der Inanspruchnahme von „Riester-Produkten“ deutlich unter $50 \%$ liegt. Der politisch gewünschte teilweise Übergang vom Zwangssparen in der GRV zu einem freiwilligen System ist auch acht Jahre nach der Einführung der Riester-Rente noch nicht einmal von der Hälfte der förderberechtigten Personen vollzogen worden. Erste empirische Studien zeigen, dass die staatliche Förderung der Riester-Rente, die gerade im unteren Einkommensbereich zu häufigerem und höherem Sparen für die Altersvorsorge führen soll, weder den Anteil der sparenden Haushalte in dieser Gruppe noch deren Sparquote erhöht (Corneo et al. 2007).
Aktuell am stärksten von der Krise betroffen sind die staatlich geförderten Fondsverträge. Zwar ist hinsichtlich der Riester-Fondssparpläne zu lesen: „Rentner und junge Riester-Sparer können beruhigt sein: Die Finanzkrise beeinflusst ihre Rente nicht - nur die Rendite" (Süddeutsche Zeitung 12.12.2008). Aber eine möglichst hohe Rendite, insbesondere im Vergleich zu jener der GRV, galt als Hauptgrund für die Einführung der staatlich geförderten PAV. Grundsätzlich sind geleistete Einzahlungen und staatliche Zulagen bei Rentenbeginn garantiert, jedoch weder die nominale noch die reale Rendite. Zudem wird die Rendite vermindert durch die Kosten für die Bewirtschaftung solcher Fonds.

Bei nicht staatlich geförderten, nicht zertifizierten Produkten zur Altersvorsorge gelten andere Regeln: Wie sicher das in einer Police angelegte Geld ist, hängt von der jeweiligen Vertragsvariante ab. Fondsgebundene und klassische Lebens- und Rentenversicherungen unterscheiden sich erheblich. Bei fondsgebundenen Verträgen trägt oft allein der Kunde das Anlagerisiko. Dabei hat eine von drei fondsgebundenen Policen keine Garantie. Wer in einer Krisenphase wie jetzt den Vertrag ausgezahlt bekommt und keine Garantie hat, muss mit Verlusten rechnen. Wer einen noch lange laufenden Vertrag hat, kann immerhin darauf hoffen, dass sich die Börsen erholen und Verluste ausgeglichen werden.

Private Pensionsfonds haben bis Ende 2008 durchschnittlich $23 \%$ ihres Wertes verloren. Der Verlust wird mit 5,4 Bill. US\$ beziffert (OECD 2009). Bei konventionellen Policen ist die Lage ganz anders. Hier muss der Versicherer bei Abschluss für die gesamte Laufzeit eine Mindestverzinsung garantieren. Bei aktuell abgeschlossenen und jüngeren Verträgen liegt diese garantierte Verzinsung bei 2,25\%, bei älteren bei bis zu $4 \%$.

\section{ZWISCHENFAZIT}

Grundsätzlich bedeutet eine Verlagerung der Altersvorsorge von der GRV hin zu betrieblicher und privater Altersvorsorge eine Übertragung der Anlagechancen wie -risiken von der Solidargemeinschaft auf die Individuen bzw. die jeweiligen Haushalte. Wichtig ist deshalb die Ausgestaltung der jeweiligen Renten, z. B. gemäß den Regeln für eine Zertifizierung der Produkte durch das Bundesamt für Finanzdienstleistungen. Private wie betriebliche Vorsorge zur
Ergänzung der staatlichen Pflichtsysteme beinhalten häufig das Problem der reduzierten Risikoabdeckung, z. B. bei vorzeitigem Ruhestand, Erwerbsminderung und Langlebigkeit.

Zusammenfassend vermuten wir, dass die Krise zu Vertrauensverlusten gegenüber auf freiwilliger Basis beruhender Altersvorsorge führt. Die begründete Befürchtung, gering regulierte Finanzmärkte könnten die eigene Altersvorsorge entwerten, wirkt sich negativ auf die Sparbereitschaft aus. Deshalb, aber auch wegen der Probleme auf dem Arbeitsmarkt, wegen der Beschäftigungsverluste und sinkenden Einkommen, müssen die Anbieter solcher Vorsorgeinstrumente mit Zahlungsunterbrechungen bzw. generell mit Absatzschwierigkeiten rechnen. Gleichzeitig zeichnet sich eine zunehmende Ungleichheit der Alterseinkünfte ab: Viele Bezieher niedriger Einkommen werden die entstehende Vorsorgelücke nicht schließen können. Auch Kurzarbeit und Arbeitslosigkeit führen zu einer geringen Inanspruchnahme zusätzlicher Altersvorsorge. Und die bereits hinreichend Versorgten profitieren von Mitnahmeeffekten der staatlichen Förderung bei betrieblicher und privater Altersvorsorge.

Angesichts dieser Situation wäre es angebracht, die Möglichkeiten zur staatlich geförderten betrieblichen und privaten $\mathrm{Al}$ tersvorsorge zu vereinfachen und gegen Inflation zu indexieren (Hauser 2009) sowie für eine adäquate Finanzierung der GRV zu sorgen (Schmähl 2000). Mehr finanzielle „Alphabetisierung" und regulierte, transparente, planbare und dynamische Produkte zur Altersvorsorge könnten helfen, die akute Vertrauenskrise zu überwinden und höhere Beteiligungsquoten zu erreichen.

\footnotetext{
8 Je nachdem, wer die Beiträge bezahlt, spricht man von arbeitgeber- oder arbeitnehmerfinanzierten Versorgungen. Mischformen sind üblich. Tendenziell steigt die Arbeitgeberseite aus der Finanzierung der betrieblichen Altersvorsorge aus. Für die Finanzierungsform "ausschließlich Arbeitnehmer" steigt der Anteil von 2001 bis 2007 von $26 \%$ auf $32 \%$, während der Anteil der Finanzierungsform "ausschließlich Arbeitgeber " von $54 \%$ auf $38 \%$ sinkt (Kortmann 2008).
} 


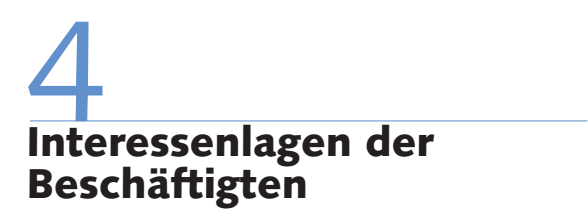

Bei den folgenden Überlegungen gehen wir davon aus, dass es keinen schnellen Ausweg aus der Krise gibt (wenn es ihn doch gibt, umso besser). Zwar hat das Statistische Bundesamt bereits ein Ende der Rezession deklariert. Die Wachstumsrate liege schon wieder im positiven Bereich. Aber die oben skizzierten Erfahrungswerte aus vergangenen Krisen legen nahe, dass alle Wirtschaftsakteure sich auf einige Jahre einstellen müssen, in denen es um die Verteilung von Verlusten bei möglichst geringer Beeinträchtigung der Zukunftsaussichten geht. Wir konzentrieren uns bei dem Versuch, Interessenlagen zu rekonstruieren, auf die abhängig Beschäftigten.

Deren wirtschaftliche Lage hat sich im Durchschnitt seit der deutschen Einheit bereits kontinuierlich verschlechtert. Die Reallöhne (Brutto) sind im Trend gesunken, sowohl pro Kopf als auch pro Stunde, nicht zu reden von den verfügbaren Realeinkommen. Die Sozialabgaben und Lohnsteuern sind seit der Einheit kräftig gewachsen (bei vorübergehender Entlastung bei den Steuern von 2003 bis 2007). So liegen die durchschnittlichen realen Netto(stunden)löhne 2008 auf dem westdeutschen Niveau von 1991. Die bereinigte Lohnquote ist seit $1982 \mathrm{im}$ Trend auf $61 \%$ gefallen, entsprechend haben die Selbstständigen- und Kapitaleinkünfte zugenommen (s.o.).

Man kann in der entstandenen Verteilungsschieflage eine Krisenursache vermuten, denn die gestiegenen Gewinne sind größtenteils nicht in Wachstum und Beschäftigung fördernde Investitionen, sondern in spekulative Anlagen auf den internationalen Finanzmärkten geflossen. Wie dem auch sei, jedenfalls haben sich die abhängig Beschäftigten von den Dauerforderungen nach Lohnzurückhaltung angesichts von Globalisierung und Arbeitslosigkeit zum eigenen Nachteil zu stark beeindrucken lassen, deutlich stärker als in fast allen anderen reichen Ländern. Die so ermöglichte Exportweltmeisterschaft hat ihnen nicht viel gebracht. Das Gesamtwachstum blieb dennoch (ebenfalls im internationalen Vergleich) extrem niedrig. Selbst die immer wieder als Erfolg der Lohnzurückhaltung behaupteten Beschäfti- gungserfolge verschwinden, wenn man sie vor dem Trend eines sinkenden Gesamtarbeitsvolumens sieht (von der Vring 2009).

Realistisch können die meisten abhängig Erwerbstätigen, sofern sie beschäftigt sind und bleiben, gegenwärtig nur ein Interesse an der Minimierung ihrer Einkommensverluste haben, gepaart mit der Hoffnung auf ein langfristig stabiles soziales Sicherungssystem. Doch selbst wenn dieses stabil bleibt, rutschen die Langzeitarbeitslosen auf Hartz-IV-Niveau. Und viele GRV-Versicherte haben unter den Bedingungen einer weiteren Erosion der Normalarbeitsverhältnisse keine ausreichende Altersversorgung mehr. Der Teil der Erwerbstätigen, der Niedriglöhne bezieht, ${ }^{9}$ hat kaum Möglichkeiten, sich zusätzlich zu staatlicher Grundsicherung Schutz vor Armut im Alter zu kaufen. Für diejenigen, die sich auf die viel gepriesene dritte Säule der Alterssicherung mit eigenen Beiträgen stützen wollten, brachte die Finanzkrise eine erhebliche Verunsicherung, wenn nicht erhebliche dauerhafte Verluste. Angesichts der akuten Krise rücken jedoch die langfristigen Probleme der Altersvorsorge in den Hintergrund, zumindest für all diejenigen, deren altersbedingtes Ausscheiden aus der Erwerbstätigkeit nicht unmittelbar bevorsteht.

Nach den letzten Jahrzehnten mit sehr schwachem Wachstum, hoher Arbeitslosigkeit und sinkenden Reallöhnen beschert die Krise zusätzliche negative Erfahrungen. Als Steuerzahler sind die abhängig Beschäftigten bei der Bankenrettung bereits zahlungspflichtig, wobei die „systemrelevanten" Banken für die Verluste aus ihren leichtfertigen spekulativen Geschäften nicht selbst gerade stehen müssen (s. die „Rettung“ der Hypo Real Estate). Was die Krise, abgesehen von den Beschäftigungsrisiken aufgrund von Insolvenzen und Stellenstreichungen, noch bringt, sind die Folgen der enorm gestiegenen und weiter steigenden Staatsverschuldung. Mit diesen Folgen ist nicht gemeint, wie der Chor der ökonomischen Naivität aus allen Parteien und Medien intoniert, dass wir "uns“ auf Kosten „unserer Kinder und Enkel“ verschulden. Die Kinder und Enkel erben nicht nur die Schulden, sondern auch die Ansprüche auf Verzinsung und Rückzahlung dieser Schulden. Es kann also, wie die Redeweise von „wir“ und „uns“ suggeriert, nicht darum gehen, dass in Fragen der Staatsverschuldung alle im gleichen Boot sitzen. Sondern es geht darum, dass ein steigender Teil der künftigen Steuereinnahmen für die Tilgung und Verzinsung der Staatsschulden verwendet werden muss. Diese Gelder fließen von den Steuerzahlerinnen auf die Konten der Eigentümer von Staats(schulden)papieren. Abgesehen von dem Umverteilungseffekt stehen sie nicht mehr für öffentliche Zwecke wie Sozialleistungen, Infrastruktur- oder Bildungsinvestitionen zur Verfügung.

Das wirft im Hinblick auf Interessen ein Entscheidungsproblem auf: Ist es wichtiger, öffentliche Leistungen, bei denen es um die zukünftigen Entwicklungschancen der Wirtschaft geht, aufrecht zu erhalten? Oder wird es als wichtiger angesehen, den weiteren Anstieg der Staatsverschuldung möglichst schnell zu bremsen? Letzteres geht nur durch höhere Steuereinnahmen oder geringere Ausgaben. Höhere Staatseinnahmen lassen sich auf zwei Wegen erzielen: entweder durch das Wachstum der Steuer- bzw. Beitragsbasis, also des Volkseinkommens, oder durch Erhöhung der Steuersätze. Das Wiedereinsetzen des Wachstums braucht Zeit, in der die Staatsverschuldung weiter wächst (erinnert sei hier an den mittleren Erfahrungswert von $86 \%$ Anstieg der Staatsverschuldung, s.o.). Demgegenüber wäre jedoch in der gegebenen Krisensituation eine Steuererhöhung Gift für eine mögliche konjunkturelle Erholung. Sie muss bei den erwartbaren induzierten Wachstumsverlusten ihr Ziel, höhere Staatseinnahmen zu generieren, verfehlen. Das ist zwar - siehe die Mehrwertsteuererhöhung nach der Bundestagswahl 2005 - offenbar kein Hinderungsgrund. Aber wahrscheinlicher als Steuererhöhungen sind dennoch Versuche, die Staatsausgaben zu kürzen.

Es gibt viele Kürzungsmöglichkeiten, aber welche sind im Interesse der abhängig Beschäftigten? Im Prinzip gar keine, jedenfalls nicht in der gegebenen Krisensituation, wo es auf die weitere Stabilisierung der effektiven Nachfrage ankommt. Selbst abgesehen von dem Deflationsrisiko, das in Deutschland offenbar gar nicht gesehen wird, ist das weitere Ansteigen der Staatsverschuldung verfrühten Kürzungsversuchen vorzuziehen. Sobald Wachstum wieder einsetzt, muss der Abbau der Staatsver-

9 Die Zahl der geringfügigen Beschäftigungsverhältnisse stieg von 4,1 Mio. 2002 auf 6,8 Mio. im März 2009 (Minijob-Zentrale 2009). 
schuldung bewerkstelligt werden, und zwar ohne den „Ausweg“ der Geldentwertung zu wählen. ${ }^{10}$

Wenn wir der Interessenlage der abhängig Beschäftigten die der politischen Akteure gegenüberstellen, wird jedoch deutlich, dass Letztere sich nicht ohne Weiteres auf die wirtschaftspolitische Variante "wachsende Staatsverschuldung zur Ermöglichung von Wirtschaftswachstum“" einlassen können. Denn einerseits gibt es keine Garantie für den Erfolg dieser Alternative, andererseits ist die Befürchtung, wachsende Staatsverschuldung bedinge Inflation, auch für die einflussreiche soziale Gruppe der Besitzer größerer Vermögen maßgeblich, die als Kreditgeber ein Interesse an einer stabilen Währung haben. Die politischen Akteure sind folglich aufgrund ihres eigenen Interesses an Machterwerb oder Machterhalt gezwungen, nicht nur die Interessen großer Wählergruppen, sondern auch die der Unternehmer und der Vermögensbesitzer zu berücksichtigen. Zwar sind weder die Interessen von Unternehmern noch die der Vermögensbesitzer einheitlich, aber man kann mit einiger Sicherheit davon ausgehen, dass es ein gemeinsames Interesse an Rentabilität und damit an Reduktion von Kostenbelastungen gibt, zu denen neben Steuern für die Unternehmen die Löhne und Sozialbeiträge (als sogenannte Lohnnebenkosten) gehören. Bei den Unternehmen kann man eine gewisse Ambivalenz in Bezug auf sozialstaatliche Sicherungen vermuten. Wie das Beispiel Kurzarbeit in der Krise zeigt, können die Unternehmen einen Teil der Krisenlasten auf den Sozialstaat umschichten. Andererseits sind auch Lohnnebenkosten Kosten, die es zu reduzieren gilt. Das gilt insbesondere unter dem Druck der Krise, bei schrumpfenden Absätzen und Gewinnen und drohender Insolvenz.

Auf jeden Fall wäre es für die zukünftigen Wachstums- und Entwicklungschancen und damit langfristig für die Beschäftigung verheerend, wenn die - im internationalen Vergleich - schon vor der Krise sehr mageren staatlichen Bildungsinvestitionen zurückgenommen würden. Daran können weder Unternehmen noch ihre Beschäftigten ein Interesse haben - gegebenenfalls aber Politiker, die kurzfristig denken und unmittelbar schmerzhafte, alternative Ausgabenkürzungen vermeiden wollen. Ebenso wäre es sowohl für den $\mathrm{Zu}$ sammenhalt der Gesellschaft als auch für die Betroffenen verheerend, wenn die so- zialen Leistungen für mehr Langzeitarbeitslose und Transferempfänger noch weiter hinter der allgemeinen Einkommensentwicklung zurückblieben, als es aufgrund vergangener Reformen bereits der Fall ist.

\section{$3_{\text {Ausblick }}$}

Die - offenbar weitgehend unter der Illusion, das Schlimmste an der Krise sei überstanden, - neu gewählte Regierung hat vier Jahre Zeit bis zur nächsten Wahl. Das Rezept wird deshalb sein: Die schmerzhaften Sachen machen wir gleich und hoffen dann auf rechtzeitige Erholung oder - falls diese ausbleibt - auf das kurze Gedächtnis des Wahlvolks. Erwartbar ist also - angesichts der bereits drastisch gestiegenen und weiter steigenden Staatsverschuldung und angesichts der ortsüblichen Angst davor - der Versuch, eine schnelle und drastische Reduktion der Staatsausgaben durchzusetzen.

Aber kann man ernsthaft hoffen, dass sich bis zum Ablauf der Wahlperiode alles wieder eingerenkt hat? Abgesehen von den Belastungen der Empfänger gekürzter Sozialleistungen wird die durch Austeritätspolitik induzierte Schwächung der Binnennachfrage die Krise verschärfen, nicht nur kurzfristig und nicht nur in ihren Auswirkungen auf die Beschäftigung. Traditionell hofft man in Deutschland in einer solchen Situation auf den Export, aber die Nachfrage aus dem Ausland ist weder berechennoch kontrollierbar, abgesehen von der Absurdität, dass die mit Lohnzurückhaltung erkauften riesigen deutschen Außenhandelsüberschüsse dann in „Finanzprodukte“ wie Lehman-Zertifikate angelegt und verpulvert wurden. Da der deutsche Exportfetischismus (Economist, 8.8.2009, S. 62) keine Lösung des Nachfrageproblems bringen wird, werden die durch Arbeitslosigkeit bedingten Ansprüche an den Fiskus steigen, bei sinkenden Einnahmen. Für gelernte Austeritätspolitiker drängt sich dann immer wieder die nächste Ausgabenkürzung auf, die folgerichtig in die gleiche, nun verschärfte Situation führt. Kurzum, zu befürchten ist, dass das Kalkül, schnelle schmerzhafte Eingriffe führen zu schneller Erholung, nicht aufgehen wird. Wegen der Schwächung der Binnennachfrage und der wahrscheinlich auch im kommenden
Zyklus für zufriedenstellendes, beschäftigungswirksames Wachstum unzureichenden Exportnachfrage droht vielmehr das Trudeln in eine Abwärtsspirale bzw. das Abgleiten in Stagnation.

Bisher geben weder die öffentliche Diskussion in den dominanten Medien noch der politische Diskurs Anlass zu hoffen, dass man in Deutschland bereit sei aus der Krise und ihren Auswirkungen auf die Systeme sozialer Sicherung zu lernen, so bspw. über das mit großem Aufwand propagierte, aber versagende Mehr-Säulen-Modell der Alterssicherung und die Leistungsfähigkeit eines kapitalgedeckten Rentensystems oder bezüglich der Arbeitslosenversicherung, für die die Beiträge bei schon anlaufender Krise noch mit großem Pomp gesenkt wurden. Wegen Fehlsteuerungen der wirtschaftspolitischen Diskurse sind die meisten Politiker noch nicht einmal in der Lage, auf die Vorzüge der automatischen Stabilisatoren hinzuweisen, die ein ausgebautes sozialstaatliches System bei Rezessionen und dem Abschmelzen der effektiven Nachfrage im Vergleich zu einem Laissez-faire-System à la USA hat. Das Vokabular klingt zu keynesianisch, also kann man sich als Anhänger neoklassischer Einfachrezepte die positiven Effekte nicht gutschreiben. Politik und staatstragende Kräfte werden deshalb versuchen, so weit wie möglich mit „business as usual“ über die Runden zu kommen.

Das aber stößt auf zwei Probleme: Erstens hat Deutschland als Exportweltmeister mit hohen Außenüberschüssen als Gegenposten zu hohen Defiziten in den USA und Teilen der Europäischen Union zu jenem globalen Ungleichgewicht beigetragen, das die Schärfe der Krise mitbedingt hat. Das altbundesrepublikanische Modell des Makromerkantilismus wird in der neuen globalen Konstellation nicht mehr funktionieren, wenn die US-Amerikaner nicht mehr auf Kredit konsumieren können. Das Modell Exportweltmeister hat aber ohnehin schon in den letzten 17 Jahren versagt. Ohne Stärkung des Binnenkonsums und - darauf folgend - der Bin-

\footnotetext{
10 Die in Deutschland gegenüber der akuten Deflationsgefahr blind machende Angst vor einer Inflation, die als Folge der Staatsverschuldung befürchtet wird, hat gegenwärtig immerhin den positiven Effekt, dass die bereits durch die Probleme der Altersvorsorge beeinträchtigte Sparneigung noch geringer wird. Man gibt sein Geld lieber aus, solange es noch etwas "wert" ist.
} 
neninvestitionen wird es keinen für Beschäftigungsstabilisierung oder -wachstum hinreichenden Wachstumsschub geben. Zweitens akzeptieren die Vermögensbesitzer und die sparenden Teile der Bevölkerung wegen Inflationsangst auf Dauer keine über Maastricht-Kriterien hinausgehende Staatsverschuldung. Ein Abbau der
Staatsverschuldung ohne kräftiges Wachstum ist jedoch nur über eine Reduzierung der Staatsausgaben (Steuererhöhungen sind Gift bei Niedrigwachstum) möglich. Für die Politik besteht also das Dilemma: entweder Kürzung von Sozialleistungen und Gefährdung des Machterhalts in der Wählergunst oder Erhöhung der Staatsver- schuldung (mindestens für die Dauer der Krise) und Gefährdung des Machterhalts vonseiten der Plutokraten. Man kann vermuten, dass Koalitionen im oder mit dem bürgerlichen Lager an diesem Dilemma zerbrechen.

\section{LITERATUR}

Bundesministerium für Arbeit und Soziales (BMAS) (2008): Statistisches Taschenbuch 2008

Brenke, K. (2009): Reallöhne in Deutschland über mehrere Jahre rückläufig, in: DIW-Wochenbericht 33, Berlin, S. 550-560

Brenke, K. (2007): Zunehmende Lohnspreizung in Deutschland, in: DIWWochenbericht 6, Berlin, S. 73-79

Bundesagentur für Arbeit (BA) (2009a): Die Entwicklung des Arbeitsund Ausbildungsstellenmarktes im Juli 2009, Presse Info 063 vom 30.07. Bundesagentur für Arbeit (BA) (2009b): Der Arbeits- und Ausbildungsmarkt in Deutschland - Monatsbericht Juli, Nürnberg

Bundesagentur für Arbeit (BA) (2009c): Bundesagentur legt Finanzbericht über das zweite Quartal 2009 vor, Presse Info 062 vom 29.07. Bundesagentur für Arbeit (BA) (2009d): Der Arbeitsmarkt in Zahlen Beschäftigungsstatistik www.pub.arbeitsamt.de/hst/services/statistik/ detail/b.html

Bundesregierung (2008): Lebenslagen in Deutschland. Dritter Armutsund Reichtumsbericht der Bundesregierung, Bundestagdrucksache 16/9915 vom 30.06

Corneo, G./Keese, M./ Schröder, C. (2007): Erhöht die Riester-Förderung die Sparneigung von Geringverdienern?, Christian-Albrechts-Universität Kiel (Hrsg.): Economics Working Paper 2007-30

Deeke, A. (2008): Konjunkturelle Kurzarbeit - Was kann bei vorübergehendem Arbeitsausfall bewirkt werden?, in: WSI-Mitteilungen 8, S. 446452

Deutsche Rentenversicherung Bund (2009): Pressemitteilung vom 23.01. Hauser, R. (2009): Vorschlag für eine inflationsindexierte Riesterrente, in: Soziale Sicherheit 5, S. 183-184

Himmelreicher, R./Stuchlik, A. (2008): Entwicklung und Verteilung von Entgeltpunkten in der gesetzlichen Rentenversicherung, in: DRV 6, S. 532-547

Kortmann, K. (2008): Situation und Entwicklung der betrieblichen Altersversorgung in Privatwirtschaft und öffentlichem Dienst 2001 - 2007, Endbericht http://www.bmas.de/coremedia/generator/30028/ property=pdf/f384_forschungsbericht.pdf
Mackenroth, G. (1952): Die Reform der Sozialpolitik durch einen deutschen Sozialplan, in: Külp, B./Schreiber, W. (Hrsg.) (1971): Soziale Sicherheit, Reihe: Neue Wissenschaftliche Bibliothek 40, Köln/Berlin, S. $265-275$

Minijob-Zentrale (2009): Aktuelle Entwicklungen im Bereich der geringfügigen Beschäftigung, I. Quartal 2009, hrsg. von: Deutsche Rentenversicherung Knappschaft-Bahn-See/Minijob-Zentrale, Essen OECD (2009): Pension at a Glance 2009. Retirement-Income Systems in OECD Countries, Paris

Reinhart, C. M./Rogoff, K. S. (2009): The Aftermath of Financial Crises, NBER Working Paper 14656, Cambridge, MA online: www.nber.org/ papers/w14656

Riedmüller, B./Willert, M. (2008): Die Zukunft der Alterssicherung. Analyse und Dokumentation der Datengrundlagen aktueller Rentenpolitik. Gutachten im Auftrag der Hans Böckler Stiftung, Düsseldorf, Mai, online: http://www.boeckler.de/pdf_fof/S-2008-90-4-1.pdf

Schaff, G. (2009): Kurzarbeit 2009: Arbeitsrechtliche, sozialversicherungsrechtliche und steuerliche Aspekte konjunktureller Kurzarbeit, hrsg. von: Arbeitnehmerkammer Bremen

Schmähl, W. (2000): Perspektiven der Alterssicherungspolitik in Deutschland: über Konzeptionen, Vorschläge und einen angestrebten Paradigmenwechsel, in: Perspektiven der Wirtschaftspolitik 4, S. 407-430 Sesselmeier, W./Haupt, M./Sommagio G./Yollu-Tok, A. (2009): Auswirkungen der Finanz- und Wirtschaftskrise auf die Soziale Sicherung, in: Sozialer Fortschritt 8, S. 183-188

Statistisches Bundesamt (2009): Gesetzliche Sozialversicherung: Defizit im ersten Quartal 2009 rückläufig, Pressemitteilung 273 vom 21.07. Viebrok, H./Himmelreicher, R./Schmähl, W. (2004): Private Vorsorge statt gesetzlicher Rente: wer gewinnt, wer verliert?, Münster Von der Vring, Th. (2009): Bilanz der Lohnzurückhaltung 2000-2007 im volkswirtschaftlichen Kreislauf Deutschlands, in: WSI-Mitteilungen 6, S. 319-323 